\title{
Randomised, clinically controlled trial of intensive geriatric rehabilitation in patients with hip fracture: subgroup analysis of patients with dementia
}

\author{
Tiina M Huusko, Pertti Karppi, Veikko Avikainen, Hannu Kautiainen, Raimo Sulkava
}

\begin{abstract}
Objective To evaluate the effect of intensive geriatric rehabilitation on demented patients with hip fracture. Design Preplanned subanalysis of randomised intervention study.

Settting Jyväskylä Central Hospital, Finland. Participants 243 independently living patients aged 65 years or older admitted to hospital with hip fracture.

Intervention After surgery patients in the intervention group $(n=120)$ were referred to the geriatric ward whereas those in the control group were discharged to local hospitals.

Main outcome measures Length of hospital stay, mortality, and place of residence three months and one year after surgery for hip fracture.

Results The median length of hospital stay of hip fracture patients with moderate dementia (mini mental state examination score 12-17) was 47 days in the intervention group $(\mathrm{n}=24)$ and 147 days in the control group $(\mathrm{n}=12, \mathrm{P}=0.04)$. The corresponding figures for patients with mild dementia (score 18-23) were 29 days in the intervention group $(\mathrm{n}=35)$ and 46.5 days in the control group $(\mathrm{n}=42, \mathrm{P}=0.002)$. Three months after the operation, in the intervention group $91 \%$ (32) of the patients with mild dementia and $63 \%$ (15) of the patients with moderate dementia were living independently. In the control group, the corresponding figures were $67 \%$ (28) and 17\% (2). There were no significant differences in mortality or in the lengths of hospital stay of severely demented patients and patients with normal mini mental state examination scores.

Conclusions Hip fracture patients with mild or moderate dementia can often return to the community if they are provided with active geriatric rehabilitation.
\end{abstract}

\section{Introduction}

The number of demented patients with hip fracture is increasing as the population ages. In Finland, the prevalence of moderate to severe dementia in people aged 65 years and over is 6.7 per $1000 .{ }^{1}$ Alzheimertype dementia has been reported to increase the risk of hip fractures, with an odds ratio of $6.9 .^{2}$ Dementia was the main contributor to the development of functional dependence and decline in a community based study of residents older than 74 years in the Kungsholmen project, Sweden. ${ }^{3}$ Dementia has also been associated with less favourable outcome of rehabilitation after hip fracture. ${ }^{4-12}$ However, selected cognitively impaired patients with hip fracture were as likely as mentally normal patients to return to the community in a specialised geriatric inpatient rehabilitation programme. ${ }^{13}$
Earlier studies provide conflicting results on the benefits of geriatric assessment and treatment in rehabilitation hospitals for elderly patients with hip fractures. ${ }^{14}$ A meta-analysis suggested that geriatric assessment programmes with intensive long term management can improve survival and function in older people. ${ }^{15}$ No randomised studies have been published on the impact of geriatric rehabilitation on demented patients with hip fracture.

Over the past decade, the median length of stay in orthopaedic wards for patients with hip fracture in central Finland healthcare district has fallen from 19 to five days, and $81 \%$ of the patients are now referred to local health centre hospitals for rehabilitation after surgery. ${ }^{16}$ Patients in local hospitals owned by local communities are treated by general practitioners. Local hospitals usually have physiotherapists, but other resources for rehabilitation vary from hospital to hospital. About half of the patients in these hospitals are in long term institutional care. In the United States the mean length of hospital stay decreased from 21.9 to 12.6 days between 1981 and $1986 .{ }^{17}$ Shorter stays were associated with less inpatient treatment and an increase in placement in long term nursing home. ${ }^{17} 18$

This study aimed to determine the effect of intensive geriatric rehabilitation after surgery for hip fracture in elderly patients. As a preplanned part of this trial, we studied whether cognitively impaired patients can benefit from geriatric assessment and intensive rehabilitation.

\section{Participants and methods}

\section{Study design}

Five per cent of the population of Finland lives within central Finland healthcare district, and Jyväskylä Central Hospital is the only place for referral for specialist care in the region. In this randomised, clinically controlled trial, community dwelling patients with acute hip fractures aged 65 years or older were randomly assigned to two rehabilitation groups after surgery at the hospital.

All patients were living independently and had been able to walk unaided before the fracture. We excluded patients with pathological fractures, multiple fractures, serious early complications, those receiving calcitonin treatment, and terminally ill patients. Fifteen patients were not able to provide consent because they had severe dementia or other serious problems with communication, and 72 patients refused to participate in the study. Twenty four patients were not asked to participate for unknown reasons, probably because the nurses on the orthopaedic ward forgot. A total of 348 patients with acute hip fractures were not randomised.

Sixteen patients were excluded because they were taking calcitonin. The patients were also randomised to nasal calcitonin or placebo for three months. The pro-

\section{Department of Rehabilitation, Division of Geriatrics, Central Hospital of Central Finland, 40930 Kinkomaa, Finland Tiina M Huusko internist and geriatrician Pertti Karppi head of geriatric division \\ Department of Orthopaedic and Trauma Surgery, Central Hospital of Central Finland, 40620 Jyväskylä, Finland \\ Veikko Avikainen head of orthopedic and trauma surgery \\ Rheumatism Foundation Hospital, 18120 Heinola, Finland Hannu Kautiainen biostatistical expert \\ Department of Public Health and General Practice, Division of \\ Geriatrics, \\ University of \\ Kuopio, PO Box 1627, 70211 Kuopio, Finland \\ Raimo Sulkava professor of geriatrics \\ Correspondence to: T M Huusko, Public Health and General Practice, University of Kuopio, PO Box 1627, 70211 \\ Kuopio, Finland Tiina.Huusko@ uku.fi}

BMJ 2000;321:1107-11 
portion of patients in these treatment groups was similar in the intervention group and in the control group.

The study was approved by the ethics committee of central Finland healthcare district, and informed consent was obtained from all participants on the first postoperative day.

All patients were encouraged to be mobile on the first postoperative day. The orthopaedic surgeons instructed the patients who had received a hemiendoprosthesis to avoid full flexion of the hip and extreme inversion or eversion depending on the technique of the operation. The patients treated by osteosynthesis were recommended to limit themselves to partial weight bearing for six weeks after the operation. It was, however, agreed that if the patient was unable to follow these recommendations he or she was allowed to be mobilised without restrictions.

\section{Intervention}

The intervention group was referred to the geriatric ward within the central hospital after randomisation, whereas the control group was discharged to local hospitals. The geriatric team at the central hospital consists of a geriatrician internist, a specially trained general practitioner, nurses with training in the care of older patients, a social worker, a neuropsychologist, an occupational therapist, and physiotherapists. A consultant specialist in physical medicine, a neurologist, and a psychiatrist work with the team for up to four days each week. The team collaborates with the patients and the families and also with the local health centres, nursing homes, home help, and home care. Common rehabilitation interventions include providing advice, training, and encouragement and listening to patients' concerns as well as drug treatment, physiotherapy, occupational therapy, speech therapy, and help with use of appliances, equipment, and daily living aids.

The intervention consisted of assessment by the geriatric team. The patients had physiotherapy sessions twice a day, and daily activities were practised throughout the day with the nurses. The nurses and the physiotherapists had weekly joint meetings to discuss methods of improving rehabilitation. The daily schedule of each patient was planned to support early ambulation, self motivation, and the best possible functional ability. The physiotherapist evaluated walking aids. An occupational therapist evaluated the need for daily living aids, and occupational therapy was offered to those patients who were thought able to benefit from it. Individualised patient and family counselling was provided by physiotherapists and nurses on several occasions, and this was reinforced with a brochure on hip fracture.

The discharge plan was checked during weekly team meetings with the patient and the family, and the physiotherapist made a home visit before the patient was discharged if the team thought that it was necessary. All patients who were discharged to independent living had 10 home visits from the physiotherapist.

\section{Assessments}

Data on living conditions and scores for the activities of daily living, ${ }^{19}{ }^{20}$ and the instrumental activities of daily living $^{21}$ at the time of the fracture were obtained from patients, relatives, home nurses, and home helps by a nurse. A doctor filled in a questionnaire on chronic morbidity, drugs, complications, and discharge plan. We studied all medical records for the first 12 months after the operation and obtained data on the lengths of stays in hospital and nursing homes, use of medical care, complications, and mortality.

The mini mental state examination ${ }^{22}$ was administered about one week after admission to the geriatric ward or the local hospital (10 days after surgery and randomisation). This examination is a well established, reliable, valid, and brief cognitive instrument that has a high interrater reliability and is easy to administer. ${ }^{23}$ Suspected severe dementia was defined as a score of $0-11$ out of a total of 30 . Suspected moderate dementia was defined as a score of 12-17, and suspected mild dementia as a score of 18-23. Scores of 24-30 were defined as normal. ${ }^{24} 25$

\section{Statistical analysis}

The principal aim of the analysis was to compare the length of hospital stay, mortality, and place of residence three months and one year after surgery for hip fracture. The results were expressed as mean or median with SD or range and $95 \%$ confidence intervals. We compared the groups using the $t$ test or Mann-Whitney $\mathrm{U}$ test. Measures with a discrete distribution were expressed as counts (\%) and analysed by $\chi^{2}$ or Fisher's exact test. We used Hommel's adjustments to correct significance levels for multiple testing. We evaluated the normality of variables by Kolmogorov-Smirnov statistics with a Lilliefors significance or Shapiro-Wilk statistics. Correlation coefficients were calculated by the Spearman method.

The planned sample size for the whole trial was about 250 (125 in each group), which gave a power of at least $80 \%$ to detect a difference of $20 \%$ between the intervention and control rehabilitation groups with two sided $\alpha=0.05$. Before the randomisation was started we had no reliable information on the number of community dwelling, demented patients who had hip fractures, but a retrospective study of the medical records of the patients admitted to the central hospital with hip fracture in 1991-3 showed that the prevalence of diagnosed dementia among patients aged 65 or older was $15 \%$ (data not shown). We assumed that a substantial difference between the intervention and the control would be detected even if the number of patients in different categories was small.

\section{Assignment and masking}

The allocation sequence was computer generated and sealed in numbered, opaque envelopes in Helsinki, Finland, by the information technology department of Novartis before the study was started. The envelopes were stored on the orthopaedic ward by the head nurse until patients were randomised.

Before the start of the study a geriatrician (TH), a nurse, and a physiotherapist visited all local hospital wards twice to clarify the objectives of the study and to teach staff how to carry out the tests and fill in the questionnaires. We could not blind the staff doing interventions or assessments. The geriatrician in charge of the study worked on another ward at the time of the study. She did not take part in geriatric rounds or team meetings. 


\section{Results}

\section{Participant flow and follow up}

From October 1994 to December 1998, a total of 260 patients with acute hip fractures were randomised (figure). Eleven patients were later excluded because of a violation of the randomisation criteria, three patients withdrew their consent after randomisation, and three patients were excluded because of a protocol violation. A total of 243 patients were followed. One patient in the intervention group and four in the control group were not tested with the mini mental state examination. The analysis was therefore conducted on 238 patients.

\section{Patients}

Table 1 shows the demographic and clinical data of the patients. Patients in the intervention group had more problems with activities of daily living and instrumental activities of daily living. The mini mental state examination scores correlated strongly with the activities of daily living scores $r=0.52$ (95\% confidence interval 0.42 to 0.61 ) and the instrumental activities of daily living scores $r=0.65$ (0.58 to 0.72$)$ at baseline.

There were $42(35 \%)$ trochanteric fractures in the intervention group and $44(36 \%)$ in the control group. All trochanteric fractures were treated by osteosynthesis. There were no differences between the intervention group and the control group in the methods of surgery for cervical fractures: $60(77 \%)$ patients in the intervention group versus $53(67 \%)$ in the control group had hemiarthroplasty, six (8\%) v 10 (13\%) received a total prosthesis, and $12(15 \%) v 16(20 \%)$ had osteosynthesis.

\section{Analysis}

The median length of stay on the geriatric ward was 18 days, after which $65(54 \%)$ patients in the intervention group were discharged to independent living. The lengths of hospital stay after surgery were calculated from the day of surgery to the day of a discharge lasting at least two weeks. Table 2 shows the lengths of hospital stay of the patients according to mini mental state examination scores. There were no significant differences between the intervention group and the control group in the length of hospital stay among patients with normal scores or among patients with scores indicating severe dementia. The median stay of patients with mild dementia was 29 days (range 16 to 138 ) in the intervention group and 46 days (range 10 to 365 ) in the control group $(\mathrm{P}=0.002)$. The median length of hospital stay of the patients with moderate dementia was 47 days (range 10 to 365 ) in the intervention group and 147 days (range 18 to 365 ) in the control group $(\mathrm{P}=0.04)$.

Table 3 shows the place of residence and the mortality of the patients three months and one year after surgery according to mini mental state examination scores. Fifteen (63\%) patients with moderate dementia in the intervention group were still living independently at three months compared with two (17\%) in the control group $(95 \%$ confidence interval of difference $11 \%$ to $69 \%, \mathrm{P}=0.009)$. Of the patients with mild dementia, $32(91 \%)$ in the intervention group were living independently at three months operation compared with $28(67 \%)$ in the control group (7\% to $42 \%$ $\mathrm{P}=0.009)$. One year after the operation, $62 \%$ of the patients with moderate dementia and $77 \%$ of the

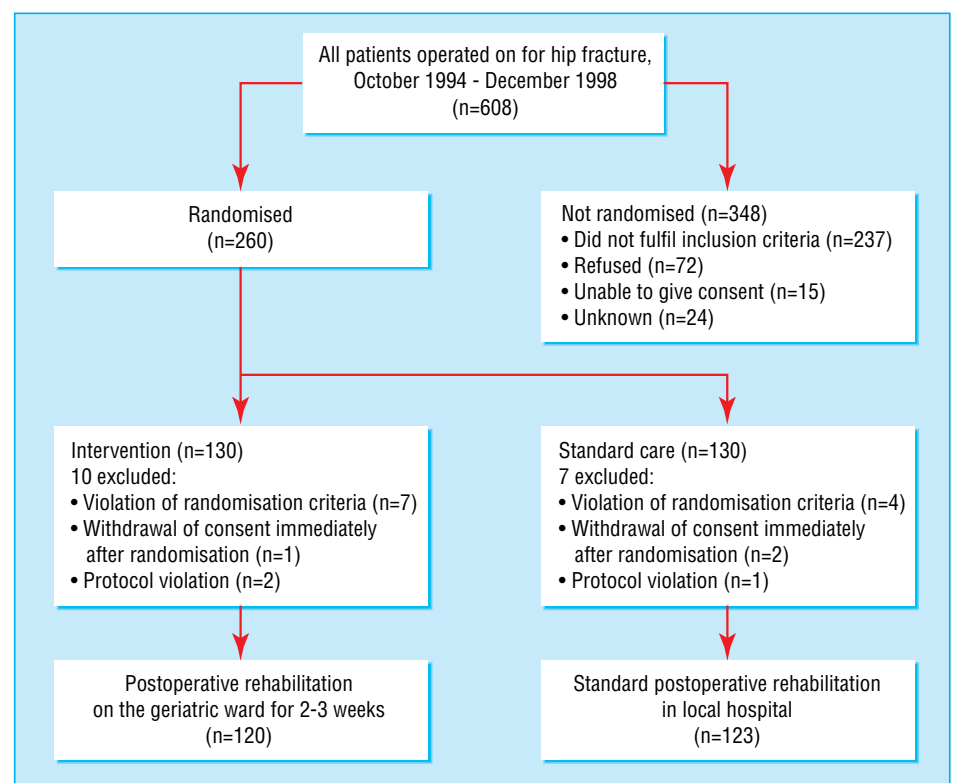

Flow of patients through study

patients with mild dementia were living independently in the intervention group. The corresponding values in the patients in the control group were 33\% $(-6 \%$ to $57 \%, \mathrm{P}=0.1)$ and $76 \%(-19 \%$ to $20 \% \mathrm{P}=0.092)$.

There were no significant differences in mortality between the intervention and the control group for any of the mini mental state groups. First year mortality for all patients in the study was $28 \%$ for patients with severe dementia, $17 \%$ for patients with moderate dementia, $10 \%$ for those with mild dementia, and $12 \%$ for patients with normal scores.

\section{Discussion}

We compared the quality of the current rehabilitation scheme in local hospitals with intensive rehabilitation in a geriatric hospital ward. Patients were recruited over four years, and the study could not have included more patients without extending to other healthcare districts and hospitals.

We found no differences in age or sex distribution, operative care, or distribution of types of hip fracture between the groups. The difference in the proportion of demented patients was coincidental. The mini mental state examination is a screening method for dementia. It has also been used to assess the severity of dementia. ${ }^{23}{ }^{24}$ We did not use any other method to

Table 1 Demographic and clinical data of the hip fracture patients in intervention and control groups

\begin{tabular}{lccc} 
& $\begin{array}{c}\text { Intervention } \\
(\mathbf{n = 1 2 0})\end{array}$ & $\begin{array}{c}\text { Control } \\
(\mathbf{n = 1 2 3})\end{array}$ & P value \\
\hline No (\%) of women & $84(70)$ & $90(73)$ & 0.58 \\
\hline Mean (range) age (years) & $80(67-92)$ & $80(66-97)$ & 0.39 \\
\hline No (\%) living alone & $62(52)$ & $70(57)$ & 0.41 \\
\hline Median (range) mini mental state examination score* & $20(0-30)$ & $23(0-30)$ & $<0.001$ \\
\hline Median (interquartile range) activities of daily living score† & $5(5-6)$ & $6(5-6)$ & 0.007 \\
\hline $\begin{array}{l}\text { Median (interquartile range) instrumental activities of daily } \\
\text { living scoreł }\end{array}$ & $4(1-7)$ & $5.5(3-8)$ & 0.004 \\
\hline Median (range) self assessed melancholy (0-100 mm) & $48(0-100)$ & $22(0-100)$ & 0.14 \\
\hline
\end{tabular}

${ }^{*}$ Full range $0-30$, higher score=better function. † Full range $0-6$, higher score=better function. ‡ Full range $0-8$, higher score=better function. IVisual analogue scale: full range $0-100$, higher score=more melancholy. 
Table 2 Length of hospital stay according to mini mental state examination score

\begin{tabular}{|c|c|c|c|c|c|}
\hline \multirow[b]{2}{*}{ Scoret } & \multicolumn{2}{|c|}{ Intervention group } & \multicolumn{2}{|c|}{ Control group } & \multirow[b]{2}{*}{$P$ value ${ }^{*}$} \\
\hline & No of patients & Median (range) stay & No of patients & Median (range) stay & \\
\hline$\overline{0-11}$ & 19 & 85 (13-365) & 9 & 67 (15-365) & 0.902 \\
\hline $12-17$ & 24 & $47(10-365)$ & 12 & $147(18-365)$ & 0.042 \\
\hline $18-23$ & 35 & $29(16-138)$ & 42 & $46(10-365)$ & 0.002 \\
\hline $24-30$ & 41 & $26(12-162)$ & 56 & $42(10-365)$ & 0.376 \\
\hline
\end{tabular}

*P value adjusted by using Hommel's method.

†Mini mental state examination was not performed on one patient in the intervention group and four in the control group.

assess cognitive impairment as the examination has been used in the health centres for many years to assess and follow up demented patients. The test was performed as soon as possible after randomisation, in a clinically stable situation. ${ }^{26}$

We found that the median lengths of hospital stay of patients with moderate or mild dementia were significantly shorter in the treatment group than in the control group, although the subgroups were small. The big difference in the median lengths of hospital stay of patients with moderate dementia was probably caused by the small number of patients in this group.

Three months after the operation, the patients in the intervention group with mild dementia were as successful as the patients with no dementia in returning to independent living. These results are comparable with the results of an earlier unrandomised study in a specialised geriatric rehabilitation programme. $^{3}$

\section{Effect of dementia}

We found that the severity of cognitive impairment was related to higher mortality and less successful return to independent living. This has been found in other studies. ${ }^{4-13}$ Lieberman and colleagues reported that the success of rehabilitation was significantly associated with reduced mental status in the mini mental state

Table 3 Place of residence and mortality of patients according to score on mini mental state examination

\begin{tabular}{|c|c|c|c|c|}
\hline \multirow[b]{2}{*}{ Score } & \multicolumn{2}{|c|}{3 months } & \multicolumn{2}{|c|}{1 year } \\
\hline & Intervention & Control & Intervention & Control \\
\hline \multicolumn{5}{|l|}{$0-11$} \\
\hline Independent living & $7(37)$ & $4(44)$ & $7(37)$ & $3(33)$ \\
\hline Nursing home & $2(11)$ & $0(0)$ & $5(26)$ & $0(0)$ \\
\hline Hospital & $8(42)$ & $4(44)$ & $2(11)$ & $3(33)$ \\
\hline Dead & $2(11)$ & $1(11)$ & $5(26)$ & $3(33)$ \\
\hline Total & $19(100)$ & $9(100)$ & $19(100)$ & $9(100)$ \\
\hline \multicolumn{5}{|l|}{$12-17$} \\
\hline Independent living & $15(63)$ & $2(17)$ & $15(62)$ & $4(33)$ \\
\hline Nursing home & $2(8)$ & $1(8)$ & $1(4)$ & $2(17)$ \\
\hline Hospital & $4(17)$ & $8(67)$ & $4(17)$ & $4(33)$ \\
\hline Dead & $3(12)$ & $1(8)$ & $4(17)$ & $2(17)$ \\
\hline Total & $24(100)$ & $12(100)$ & $24(100)$ & $12(100)$ \\
\hline \multicolumn{5}{|l|}{ 18-23 } \\
\hline Independent living & $32(91)$ & $28(67)$ & $27(77)$ & $32(76)$ \\
\hline Nursing home & $0(0)$ & $2(5)$ & $2(6)$ & $3(7)$ \\
\hline Hospital & $2(6)$ & $10(24)$ & $2(6)$ & $2(5)$ \\
\hline Dead & $1(3)$ & $2(5)$ & $4(11)$ & $5(12)$ \\
\hline Total & $35(100)$ & $42(100)$ & $35(100)$ & $42(100)$ \\
\hline \multicolumn{5}{|l|}{$24-30$} \\
\hline Independent living & $35(85)$ & $47(84)$ & $34(83)$ & $47(84)$ \\
\hline Nursing home & $0(0)$ & $1(2)$ & $1(2)$ & $1(2)$ \\
\hline Hospital & $5(12)$ & $7(12)$ & $2(5)$ & $0(0)$ \\
\hline Dead & $1(2)$ & $1(2)$ & $4(10)$ & $8(14)$ \\
\hline Total & $41(100)$ & $46(100)$ & $41(100)$ & $56(100)$ \\
\hline
\end{tabular}

examination. ${ }^{6}$ Cognitive function was assessed during the first week after admission to the rehabilitation unit. The odds of successful rehabilitation in patients without dementia were found to be 20 times higher than for a patient with dementia. The average duration of stay in a geriatric ward of patients with dementia was 34.8 days. $^{6}$

Earlier randomised studies of geriatric rehabilitation after hip fracture do not allow direct conclusions to be drawn about its effect on patients with dementia. Studies in the United States and Scotland found no significant gains in mortality or length of hospital stay with evaluation by a geriatric team and geriatric consultation on an orthopaedic ward. ${ }^{28}{ }^{29}$ The cognitive function of the patients in these studies was not reported. Kennie et al found that women with hip fracture who were admitted to a geriatric ward after surgery had a shorter median stay and were less likely to be discharged into institutional care than women who remained on an orthopaedic ward. ${ }^{30}$ After one year the mortality was lower and more patients were living independently. ${ }^{31}$ However, the patients in the control group were older and had more severe cognitive impairment than those in the intervention group. Among patients with moderate impairment, the median length of stay in hospital was 21 days in the geriatric treatment group $(\mathrm{n}=7)$ and 61 days in the control group $(n=16){ }^{30}$ Our results are comparable with these findings.

Patients in an Australian study benefited from early geriatric assessment and discharge planning, accelerated rehabilitation, and closer family caregiver contact. $^{32}{ }^{33}$ The mean length of hospital stay was shorter and more patients were discharged to independent living. The proportion of cognitively impaired patients was similar in the intervention and control groups, but the patients in the intervention group were younger than those in the control group. A Swedish study found no significant differences between rehabilitation of hip fracture patients on an orthopedic ward and rehabilitation on a local hospital ward for geriatric patients with orthopaedic problems. ${ }^{34}$ However, the cognitive function of the patients was not evaluated, the men in the intervention group were older, and there were more patients with subtrochanteric fractures in the geriatric rehabilitation group.

The quality and cost of treatment schemes depend on the surgical and rehabilitation methods and how the community cares for elderly people. Successful rehabilitation requires caring for the physical, social, and psychosocial needs of elderly patients. Demented patients with hip fractures probably benefited especially from practising daily activities throughout the day, early mobilisation and discharge, physiotherapy 


\section{What is already known on this topic}

Demented patients with hip fracture have higher mortality and are more likely to need long term residential care than patients without dementia

\section{What this study adds}

Geriatric assessment and intensive rehabilitation after hip fracture in patients with mild or moderate dementia diminishes the length of hospital stay

Patients with mild dementia in the geriatric rehabilitation group were as successful as patients with normal cognitive function in returning to independent living

One year after hip fracture, significantly fewer patients with moderate dementia in the geriatric rehabilitation group were in institutional care

Intensive, multidisciplinary geriatric rehabilitation should be considered for hip fracture patients with mild or moderate dementia

in the familiar surroundings at home, individualised family counselling, and collaboration with the families and home care.

We thank Sari Pietikäinen for acting as the research nurse and the medical and nursing staff of the geriatric and the orthopaedic wards of Jyväskylä Central Hospital and of the local hospital wards, whose cooperation was essential.

Contributors: RS planned the investigation together with TMH and VA. TMH analysed and interpreted the data, wrote the drafts of the manuscript, and acted as clinical investigator. PK acted as physician and clinical investigator and revised the manuscript. VA acted as physician and revised the manuscript HK took part in the analysing and interpreting the data. He cowrote and revised the manuscript. RS designed the study, interpreted the data, cowrote the article, and revised the drafts as well as the final version of the manuscript. He is the study guarantor.

Funding: The study was supported by grants from central Finland healthcare district, Kuopio University Hospital, Emil Aaltonen Foundation, Uulo Arhio Foundation, and Novartis Finland.

Competing interests: None declared.

1 Sulkava R, Wikström J, Aromaa A, Raitasalo R, Lehtinen V, Lahtela K, et al. Prevalence of severe dementia in Finland. Neurology 1985:35:1025-9.

2 Buchner DM, Larson EB. Falls and fractures in patients with the Alzheimer's type dementia. JAMA 1987;257:1492-5.

3 Agüero-Torres H, Fratiglioni L, Guo Z, Viitanen M, von Strauss F, Winblad B. Dementia is the major cause of functional dependence in the elderly: 3-year follow-up data from a population-based study. Am J Public Health 1998;88:1452-6.

4 Kyo T, Takaoka K, Ono K. Femoral neck fracture. Factors related to ambulation and prognosis. Clin Orthop 1993;292:215-22.

5 Parker MI, Palmer CR. Prediction of rehabilitation after hip fracture. Age Ageing 1995;24:96-8.

6 Lieberman D, Fried V, Castel H, Weitzmann S, Lowenthal MN, Galinsky D. Factors related to successful rehabilitation after hip fracture: case-control study. Disabil Rehabil 1996;5:224-30

7 Steiner JF, Kramer AM, Eilertsen TB, Kowalsky JC. Development and validation of a clinical prediction rule for prolonged nursing home residence after hip fracture. J Am Geriatr Soc 1997;45:1510-4.

8 Lyons AR. Clinical outcomes and treatment of hip fracture. Am J Med 1997; 103:51-64.

9 Heruti RJ, Lusky A, Barell V, Ohry A, Adunsky A. Cognitive status at admission: does it affect the rehabilitation outcome of elderly patients with hip fracture? Arch Phys Med Rehabil 1999;80:432-6.

10 Clayer MT, Bauze RJ. Morbidity and mortality following fractures of the femoral neck and trochanteric region: Analysis of risk factors. J Traum $1989 ; 29: 1673-8$

11 Pitto RP. The mortality and social prognosis of hip fractures. A prospective multifactorial study. Int Orthop 1994;18:109-13.
12 Van der Sluijs JA, Walenkamp G. How predictable is rehabilitation after hip fracture? A prospective study of 134 patients. Acta Orthop Scand 1991;62:567-72.

13 Goldstein FC, Strasser DC, Woodard JL, Roberts VJ. Functional outcome of cognitively impaired hip fracture patients on a geriatric rehabilitation unit. JAm Geriatr Soc 1997; 45:35-42.

14 Kramer AM, Steiner JF, Schlenker RE, Eilertsen TB, Hrincevich CA, Tropea DA, et al. Outcomes and costs after hip fracture and stroke. A comparison of rehabilitation settings. JAMA 1997;277:396-404

15 Stuck AE, Siu AL, Wieland GD, Adams J, Rubenstein LZ. Comprehensive geriatric assessment: a meta-analysis of controlled trials. Lance 1993;342:1032-6.

16 Huusko T, Karppi P, Avikainen V, Kautiainen H, Sulkava R. Significant changes in the surgical methods and length of hospital stay of hip fracture patients occurring over 10 years in central Finland. Ann Chir Gynacol 1999;88:55-60.

17 Fitzgerald JF, Moore PS, Dittus RS. Changing patterns of hip fracture care before and after implementation of the prospective payment system. N Engl J Med 1988;319:1395-7.

18 Kahn KL, Keeler EB, Sherwood MJ. Comparing outcomes of care before and after implementation of the DRG-based prospective payment system. JAMA 1990:264:1984-8.

19 Katz S, Ford AB, Moskowitz RW, Jackson BA, Jaffe MW. Studies of illness in the aged. The index of ADL: a standardised measure of biological and psychosocial function. JAMA 1963;185:914-9.

20 Katz S, Downs TD, Cash HR, Grotz RC. Progress in the development of the index of ADL. Gerontologist 1970;1:20-30.

21 Lawton MP, Brody EM. Assessment of older people: self-maintaining and instrumental activities of daily living. Gerontologist 1979;9:179-86.

22 Folstein MF, Folstein SE, McHugh PR. Mini-mental state: a practical method for grading the cognitive stage of patients for the clinician. J Psychiatr Res 1975;12:189-98.

23 Grace J, Nadler JD, White DA, Guilmette TJ, Giuliano AJ, Monsch AU, et al. Folstein vs modified mini-mental state examination in geriatric stroke Stability, validity, and screening utility. Arch Neurol 1995;52:477-84.

24 Forsell Y, Fratiglioni L, Grut M, Viitanen M, Winblad B. Clinical staging of dementia in a population survey: comparison of DSM-III-R and the Washington University Clinical Rating Scale. Acta Psychiatr Scand 1992;86:49-54

25 Hux MJ, O’Brien BJ, Iskedjian M, Goeree R, Gagnon M, Gauthier S. Relation between severity of Alzheimer's disease and costs of caring. CMA 1998;159:457-65

26 Williams M, Campbell E, Raynor WJ, Mlynarczyk SM, Ward SE. Reducing acute confusional states in elderly patients with hip fractures. Res Nur Health 1985;8:329-37.

27 Gustafson Y, Brännström B, Berggren D, Ragnarsson JI, Sigaard J, Bucht $\mathrm{G}$, et al. A geriatric-anesthesiologic program to reduce acute confusional states in elderly patients treated for femoral neck fractures. J Am Geriat Soc 1991;39:655-62.

28 Jette A, Harris BA, Cleary PD, Campion EW. Functional recovery after hip fracture. Arch Phys Med Rehabil 1987;68:735-40.

29 Gilchrist WJ, Newman RJ, Hamblen DL, Williams BO. Prospective randomised study of an orthopaedic geriatric inpatient service. $B M J$ 1988:297:1116-8

30 Kennie DC, Reid J, Richardson IR, Kiamari AA, Kelt C. Effectiveness of geriatric rehabilitative care after fractures of the proximal femur in elderly women: a randomised clinical trial. BMJ 1988;287:1083-986.

31 Reid J, Kennie DC. Geriatric rehabilitative care after fractures of the proximal femur: one year follow up of a randomised clinical trial. $B M$ 1989;299:25-6.

32 Cameron ID, Lyle DM, Quine S. Accelerated rehabilitation after proxima femoral fracture: a randomised controlled trial. Disabil Rehabil 1993;15:29-34

33 Cameron ID, Lyle DM, Quine S. Cost effectiveness of accelerated rehabilitation after proximal femoral fracture. J Clin Epidemio $1994 ; 47: 1307-13$

34 Galvard H, Samuelsson S-M. Orthopedic or geriatric rehabilitation of hip fracture patients: a prospective, randomised, clinically controlled study in Malmö, Sweden. Aging Clin Exp Res 1995;7:11-6.

(Accepted 10 August 2000)

\section{Endpiece \\ Just worn out}

I could not ascertain by my own observation, or through the medical attendants, that Sir Hildebrand Osbaldistone died of any formed complaint bearing a name in the science of medicine. He seemed completely worn out and broken down by fatigue of body and distress of mind; just as a vessel, buffeted and tossed by a succession of tempestuous gales, her timbers overstrained, and her joints loosened, will sometimes spring a leak and founder, where there are no apparent causes for her destruction.

Scott W. Rob Roy. London: J M Dent, 1908.

Submitted by Simon Barley, Sheffield 